\title{
MINIMIZATION OF METABOLIC COST OF MUSCLES BASED ON HUMAN EXOSKELETON MODELING: A SIMULATION
}

\author{
Harita Baskar and Sri Madhava Raja Nadaradjane \\ Department of Electronics and Instrumentation, St. Joseph's College of Engineering, \\ Anna University, Chennai, India
}

\begin{abstract}
In this work, movement of the exoskeleton wearer and the metabolic energy changes with the assisted devices using OpenSim platform has been attempted. Two musculoskeletal models, one with torsional ankle spring and the other with bi-articular path spring are subjected to forward dynamic simulation.The changes in the metabolic rate of the lower extremity muscles before and after the addition of the assistive devices were tested. The results about the effect of these external devices on individual muscles of the lower muscle group were analysed which provided effective results.
\end{abstract}

\section{KEYWORDS}

Human Exoskeleton, Metabolic Cost, Gastrocnemius muscle, Soleus muscle \& Illiopsoas muscle

\section{INTRODUCTION}

An exoskeleton is a particular kind of mechanical device designed to be worn by an operator that help in assisting limb movement and the execution of a motor task [1]. These exoskeletons are widely used for various orthopaedic disorders, different levels of paralysis, military applications and in rehabilitation centres [2]. The main target of this technology is to augment the human body and its capabilities[3].

Exoskeletons have the potential to reduce the metabolic cost of walking, loaded walking as well as running. Figure1 shows the main components of an exoskeleton on a loaded subject. It includes hip springs, adduction springs and an ankle spring. These spring components help in reducing the metabolic energy of the muscles. The knee damper helps in limiting the range of motion [4]. The reduction in metabolic cost decreases the possibilities of injury and increases the load carrying capacity [5]. Researchers have attempted to achieve this by developing both active and passive exoskeletons. Kazerooni, Racine, et. al. developed a lower extremity exoskeleton for load carriage with actuated hip, knee and ankle joints which described an autonomous model [6].Walsh $e t$. al. designed an quasi passive leg exoskeleton with only ankle and hip springs with a knee variable damper [7]. These devices try to assist human gait but there are certain challenges associated with such devices. It is difficult to analyse the effectiveness of the device and also cannot predict how external actuation assists muscles during loaded walking, Simulation can help develop the wearable device, as it can give an idea on how the device helps muscles and how the metabolic cost changes during loaded walking. Muscle driven simulation allows the calculation of muscle forces, residual forces, fiber length and other parameters that cannot be easily measured. It is very important to measure these neuromuscular quantities, as they are responsible for the production of movement [8]. It also makes it possible to measure the metabolic cost of different muscle groups during the gait cycle.

DOI: 10.5121/ijbes.2016.3401 
In order to achieve a simulation based design there are certain tools such as AnyBody modelling system [9], SimTK[10], Virtual Interactive Musculoskeletal System (VIMS)[11],SIMM (Software for Interactive Musculoskeletal Modeling)[12] and OpenSim[13]. Dominic et al studied the effect of elastic ankle exoskeleton on bilateral hopping by utilizing simulation of muscle tendon dynamics with a generic musculoskeletal model[14]. Recently, Yoshiaki et al evaluated the effectiveness of powered exoskeleton using computer simulation that aimed to decrease the metabolic energy consumption for upper arm reaching movement [15].

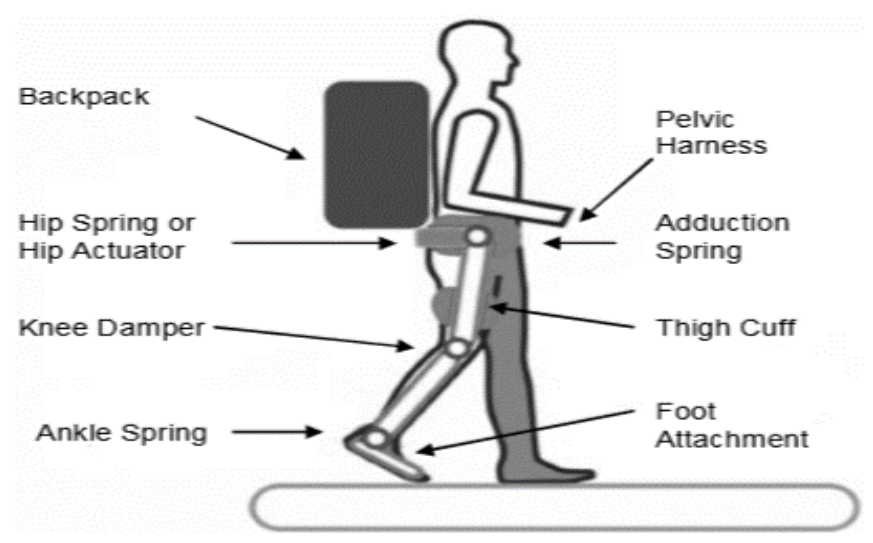

Figure 1.Components sketch of the main components of the exoskeleton

This paper focuses on the simulation of movement of the exoskeleton wearer using Open Sim platform and the metabolic energy changes associated with the lower extremity muscle group. This exoskeleton is based on the spring like action of leg and from the moment angle analysis of human stance during walking, it is seen that there exist a linear relationship among the dorsiflexion and plantar-flexion stages of the stance Therefore, the metabolic cost of the ankle joint is reduced by replacing it with a torsional ankle spring. In conjunction with a damping mechanism, the spring is able to minimize the energy expenditure during walking by storing energy during the progression stage and allowing free movement in rest of the gait cycle [16]. Forward dynamic simulation is performed for two musculoskeletal models, one with torsional ankle spring and the other with bi-articular path spring. The changes in the total metabolic cost of the lower extremity muscles after and before the addition of the assistive devices were examined. Moreover, the effects of these external devices on Gastrocnemius muscle, Soleus muscle and Illiopsoas muscle were also analysed.

\section{METHODOLOGY}

In this paper, two three-dimensional musculoskeletal models with 10 degrees of freedom with 18 muscle tendon paths with actuators were created using OpenSim. Both the models represent a male of $75(\mathrm{Kg})$ mass and $1.8(\mathrm{~m})$ height. The dynamic musculoskeletal model is used as the input for the forward dynamic simulation.. The model consist of head, trunk, pelvis, both femur, tibia and foot segments [17]. The two models are subjected to three processes before the addition of the assistive devices which includes scaling, inverse kinematics and computes muscle control. These three processes were used to create simulations of the subject running at a speed of $1.3(\mathrm{~m} / \mathrm{s})$. Then a torsional ankle spring is attached to one model and a bi-articular path spring to the other model. 


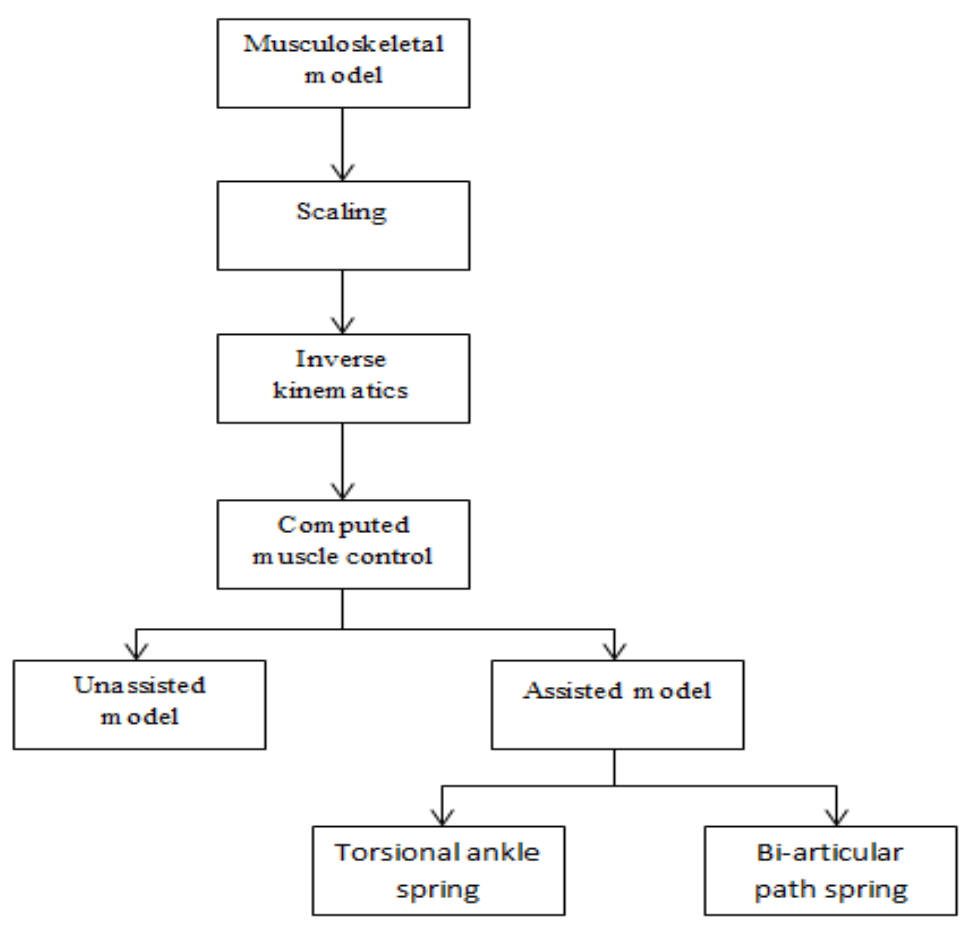

\subsection{Scaling}

Figure 2 Flowchart for dynamic simulation

The initial step to perform forward dynamic simulation is to scale a generic model to fit a particular data. It is scaled to match the required size and weight of the model. By using motion capture equipment, the marker locations are identified and this represents the experimental markers. The unscaled model already has a set of virtual markers around the same location as the experimental markers. The dimensions of each segment in the model are scaled by moving the virtual markers such that they coincide with the experimental marker locations [18].

\subsection{Inverse kinematics}

Inverse kinematics is performed to determine the generalized coordinates which represents the joint angles and translations of the model and to minimize the coordinate and marker errors . The IK tool in OpenSim ensures that the model is placed in a pose that best matches the experimental marker locations in each time frame. This is achieved through solving the weighted least square problem and the solution is mathematically expressed as

$$
q^{\min }\left[\sum_{i=1}^{\text {markers }} w_{i}\left\|x_{i}^{\text {exp }}-x_{i}(q)\right\|^{2}+\sum_{j=1}^{u n p r e s c r i b e d ~ c o o r d i n a t e s} \omega_{i}\left(q_{j}^{\text {exp }}-q_{j}\right)^{2}\right](1)
$$

Where $\mathrm{q}$ is the vector of generalized coordinates, $\mathrm{x}_{\mathrm{i}}{ }^{\mathrm{exp}}$ is the experimental position of marker $\mathrm{i}$, $\mathrm{x}_{\mathrm{i}}(\mathrm{q})$ is the position of the corresponding marker on the model (which depends on the coordinate values), $\mathrm{q}_{\mathrm{i}}^{\text {exp }}$ is the experimental value for coordinate $\mathrm{j}[19]$. The weighted square error that has to be minimized is given as 
Squared error $=\sum_{i=1}^{\text {markers }} \omega_{i}\left(\vec{x}_{i}^{\text {subject }}-\vec{x}_{i}^{\text {model }}\right)^{2}+\sum_{j=1}^{\text {joint angles }} \omega_{j}\left(\theta_{j}^{\text {subject }}-\theta_{j}^{\text {model }}\right)^{2}$

Where $\vec{x}_{i}^{\text {subejct }}$ and $\vec{x}_{i}^{\text {model }}$ are the three dimensional position of the $i$ th marker for the model, $\theta_{j}{ }^{\text {subject }}$ and $\theta_{j}^{\text {model }}$ are the values of the $j$ th joint angles for the model and $\omega_{i}$ and $\omega_{j}$ are the weight factors [13].

\subsection{Computed muscle control}

Computed muscle control (CMC) is used to generate a set of muscle excitation that drives the generalized coordinates to closely track the desired kinematics. Before using the CMC tool, the initial condition values which include the values of generalized velocities, generalized coordinates and muscle activation length have to be applied for the first 0.03 seconds of the desired interval. The forward dynamic simulation is attained by the CMC tool with the combination of static optimization and a proportional derivative control.Static optimization is used as an actuator controller to achieve the desired accelerations and helps in eliminating any noise in the data when a there is a sudden difference in acceleration during the gait cycle. The desired accelerations are computed based on the proportional-derivative control law that is written as

$$
\ddot{q}^{\exp }(t+\Delta t)=\ddot{q}^{\exp }(t+\Delta t)+K_{v}\left[\dot{q}^{\exp }(t)-q(t)\right]+K_{p}\left[q^{\exp }(t)-q(t)\right]
$$

Where $k_{v}$ and $k_{p}$ are the feedback gains on the velocity and position errors, $\ddot{q}^{\text {exp }}$ represents the desired accelerations whereas $q$ and $q^{\exp }$ represent the model coordinates and experimentally derived coordinates respectively[19].CMC is carried out until the desired movement is achieved.

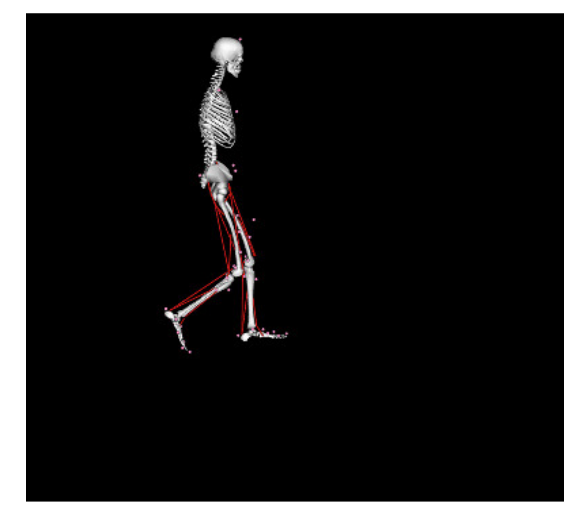

\subsection{Simulation with assistive devices}

Figure 3 Model without assistance

After the completion of the above processes, forward dynamic simulation is performed with two assistive devices: torsional ankle spring and bi- articular path spring. The torsional ankle spring is modelled at the right ankle joint and the bi-articular path spring acts long the right femur and foot segment. Both the springs act as a force element and functions as a spring and damper with a specified stiffness value. The stiffness value for the ankle spring and bi-articular path spring is set as $10(\mathrm{Nm})$ and $10000(\mathrm{Nm})$ respectively and damping ratio is 0.01 . The two force elements are assigned as a Coordinate Limit Force and this limits the range of motion when the dorsiflexion angle exceeds a specified angle(set as 5 degrees). Thus two simulations are executed with each of the springs modelled individually. 


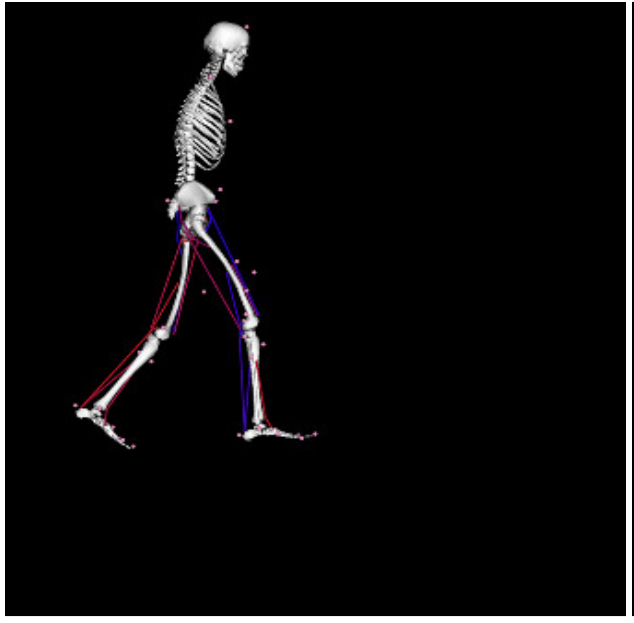

(a)

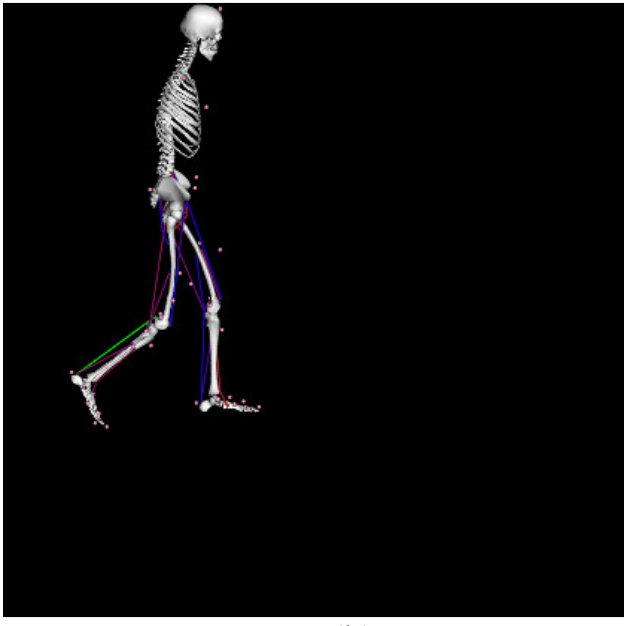

(b)

Figure 3. (a) Model With Torsional Ankle Spring, Blue Markers Identify The Connection Points (b) Model With Bi-Articular Path Spring, Green Marker Identifies The Connection Points.

\section{RESULTS AND DISCUSSIONS}

\subsection{Without assistance}

Computed muscle control (CMC) was used to generate muscle driven simulation for model without any assistance. After the application of computed muscle control, the total metabolic energy was calculatedusing the metabolic cost calculator at various time intervals. Figure 4 illustrates the total metabolic energy, which ranges from 500J-2000J. The maximum value of metabolic energy is 1852.54 (Joules) at time 1.35 seconds.

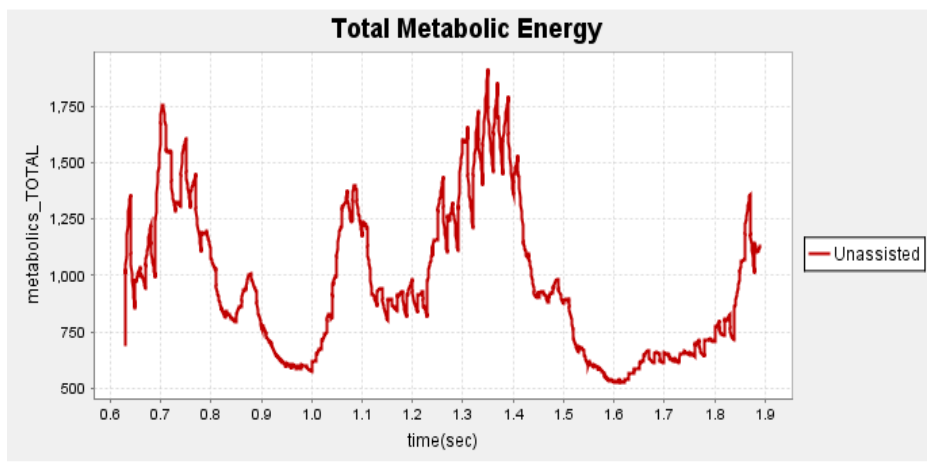

Figure 4. Total Metabolic Energy Vs Time For Unassisted Model 


\subsection{With torsional ankle spring}

A torsional ankle spring is added to the model by using the OpenSim tool. This aids in reducing the total metabolic energy of the lower extremity. The blue curve in fig 2 indicates the total metabolic energy after adding the torsional ankle spring, which shows a 30\% decrease in the total energy when compared to the unassisted model. The maximum value of total energy is 1255.18(Joules) at time 0.7 (sec).

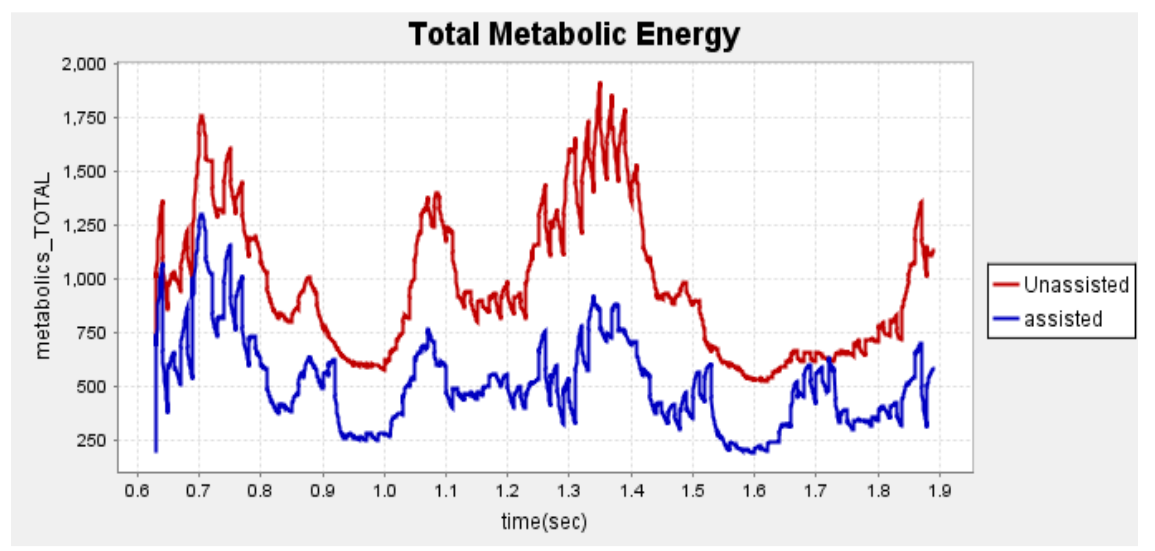

Figure 5. Comparison of Total Metabolic Energy vs Time For Unassisted

\subsection{With Bi-articular path spring}

This spring reduces the metabolic energy of the muscles along the femur and the foot segments. It significantly reduces the metabolic energy of the gastrocnemius muscle, illiopsoas muscle and soleus muscle. Figure 6 indicates that there is a 30\% decrease in the total metabolic energy when compared with the metabolic energy generated by the unassisted model. The maximum value total metabolic energy is 1252.14 (Joules) at 0.7 (seconds) of the gait cycle.

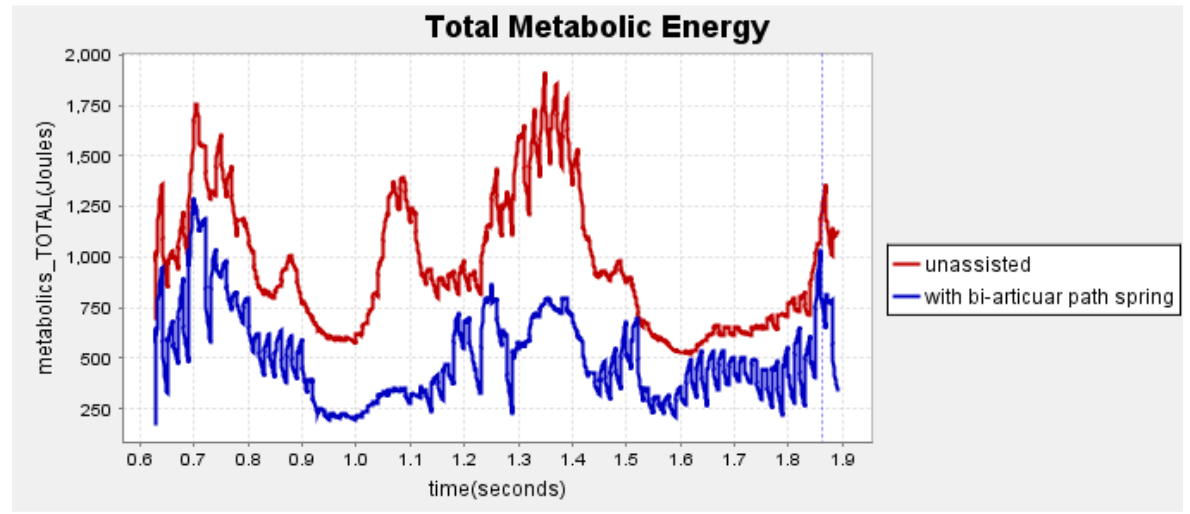

Figure 6. Total metabolic energy vs time for a model with bi-articular path spring 
Table 1 Comparison of total metabolic energy of unassisted model with assisted models with respect to time

\begin{tabular}{|c|c|c|c|c|}
\hline S.No & \multirow{2}{*}{$\begin{array}{c}\text { Time } \\
\text { (sec) }\end{array}$} & \multicolumn{3}{|c|}{ Total metabolic energy(Joules) } \\
\cline { 3 - 5 } & & $\begin{array}{c}\text { Unassisted } \\
\text { model }\end{array}$ & $\begin{array}{c}\text { Model with } \\
\text { Torsional } \\
\text { ankle spring }\end{array}$ & $\begin{array}{c}\text { Model with } \\
\text { Bi-articular } \\
\text { path spring }\end{array}$ \\
\hline 1. & 0.7 & 1752.10 & 1255.18 & 1252.14 \\
\hline 2. & 0.8 & 1101.29 & 772.19 & 662.73 \\
\hline 3. & 0.9 & 592.89 & 656.71 & 543.92 \\
\hline 4. & 1.0 & 231.42 & 110.32 & 201.15 \\
\hline 5. & 1.1 & 361.14 & 301.11 & 431.29 \\
\hline 6. & 1.2 & 701.91 & 737.81 & 744.56 \\
\hline 7. & 1.3 & 553.12 & 624.43 & 251.03 \\
\hline 8. & 1.35 & 1852.54 & 901.73 & 826.15 \\
\hline 9. & 1.4 & 761.22 & 770.10 & 669.45 \\
\hline
\end{tabular}

\subsection{Effect of Bi-articular path spring on the lower extremity muscles:}

The spring helps in reducing the metabolic energy of the Gastrocnemius muscle, Soleus muscle and Illiopsoas muscle. By using the OpenSim tool, the total metabolic energy with respect to time was obtained. From the simulation plots, the average of the total metabolic energy is calculated for each muscle group before and after the addition of the Bi-articular path spring. After the comparison of the results, it is seen that there is a substantial decrease in the metabolic energy after the addition of the Bi-articular path spring. The average value of metabolic energy for Gastrocnemius muscle is 95.487 (Joules), for Soleus muscle it is 46.604(Joules) and for Illiopsoas muscle it is 50.743(Joules).

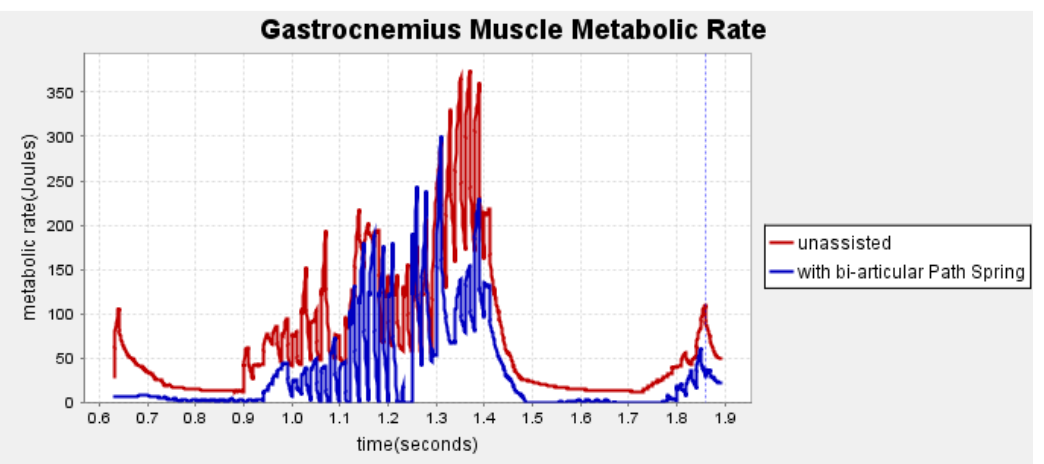

(a) 


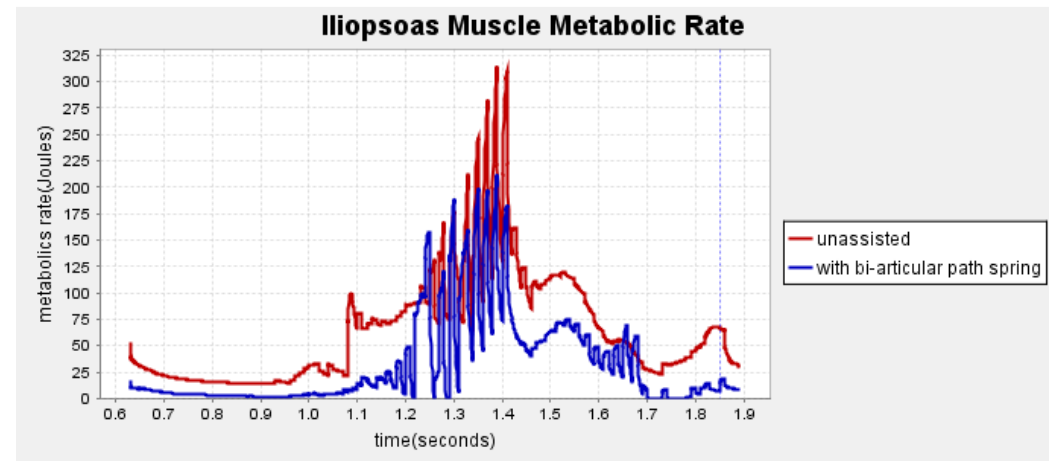

(b)

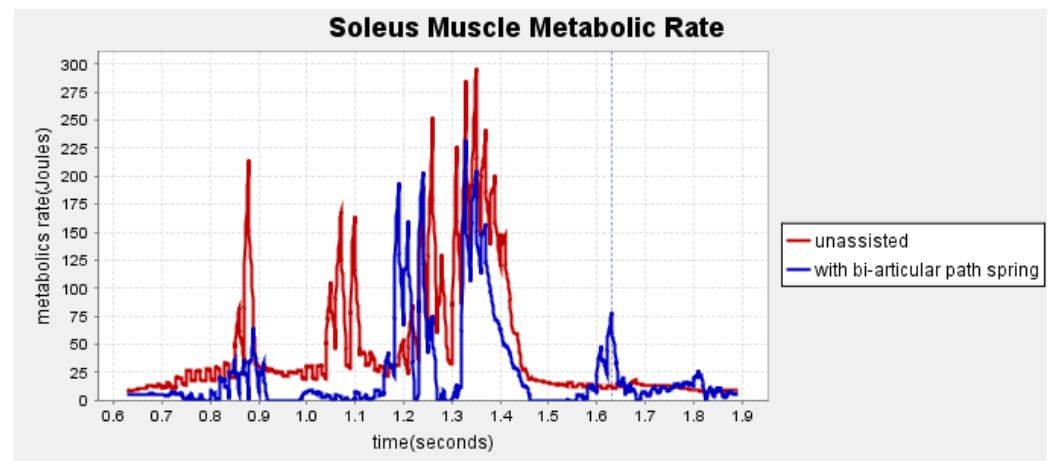

(c)

Figure 7. (a) Gastrocnemius muscle metabolic rate (b) Illiopsaos muscle metabolic rate (c) Soleus muscle metabolic rate

Table 2 Average value of metabolic energy with and without Bi-articular path spring

\begin{tabular}{|l|c|c|}
\hline \multirow{2}{*}{ Muscle group } & \multicolumn{2}{|c|}{ Metabolic energy (Joules) } \\
\cline { 2 - 3 } & $\begin{array}{c}\text { Without bi-articular } \\
\text { spring }\end{array}$ & $\begin{array}{c}\text { With bi-articular } \\
\text { spring }\end{array}$ \\
\hline $\begin{array}{l}\text { Gastrocnemius } \\
\text { muscle }\end{array}$ & 102.71 & 67.48 \\
\hline $\begin{array}{l}\text { Soleus } \\
\text { Muscle }\end{array}$ & 98.16 & 46.60 \\
\hline $\begin{array}{l}\text { Illiopsoas } \\
\text { Muscle }\end{array}$ & 88.760 & 50.74 \\
\hline
\end{tabular}

\section{CONCLUSION}

In this paper, the modelling of an exoskeleton with two assisted devices and the simulation of the exoskeleton wearer has been executed. The total metabolic energy for different muscle groups were compared with and without the assisted devices. It was examined, that me metabolic energy of each muscle has reduced with the addition of a torsional ankle spring and bi-articular path spring. For future work, these simulations can aid in evaluating the performance of powered prosthetic legs and can provide inputs for alternate prosthesis design. Furthermore, the analysis of metabolic cost of the lower extremity muscles (Gastrocnemius and soleus muscle) can forma foundation for minimizing the effects of osteoporosis and medial calf injuries. 
International Journal of Biomedical Engineering and Science (IJBES), Vol. 3, No. 4, October 2016

\section{REFERENCES}

[1] A. Moubarak, M. Tu Pham, T. Pajdla and T. Redarce, (2009), "Design and Modeling of an Upper Extremity Exoskeleton", 11th International Congress of the IUPESM : medical physics and biomedical engineering world congress 2009, Springer.pp.476-479.

[2] J. C. Perry, J. Rosen and S. Burns, (2007),"Upper-Limb Powered Exoskeleton Design",IEEE/ASME TRANSACTIONS ON MECHATRONICS, vol. 12, no. 4.

[3] J. E. Pratt, B. T. Krupp and C. J. Morse, (2004),"The RoboKnee: An Exoskeleton for Enhancing Strength and Endurance During Walking", Proceedings of the 2004 IEEE International conference on Robotics and Automation.

[4] C. James Walsh, D. Paluska, K. Pasch, W. Grand, A. Valiente and H. Herr, (2006),"Development of a lightweight, underactuated exoskeleton for load-carrying augmentation", Proceedings of the 2006 IEEE International Conference on Robotics and Automation.

[5] L. M Mooney, E. J Rouse and H. M Her, (2014),"Autonomous exoskeleton reduces metabolic cost of human walking during load carriage", Journal of NeuroEngineering and Rehabilitation, pp. 11:80.

[6] A. B. Zoss and A. Chu, (2005), "On the Mechanical Design of the Berkeley Lower Extremity Exoskeleton (BLEEX)", IEEE/RSJ International Conference on Intelligent Robot and Systems.

[7] C. James Walsh, K. Endo and H. Herr, (2007), "Design of a Quasi-Passive Parallel Leg Exoskeleton to Augment Load Carrying for Walking", International Journal of Humanoid Robotics, vol. 4, no. 3, pp. 487-506.

[8] P. Agarwal, M. S. Narayanan, L. Lee, F. Mendel and V. N. Krovi, (2010), "Simulation-Based Design of Exoskeletons Using Musculoskeletal Analysis", International Design Engineering Technical Conferences and Computers and Information in Engineering Conference.

[9] A.Technology, "AnyBody Technology", Anybodytech.com, (2016), [Online]. Available: http://www.anybodytech.com [Accessed: 03- Jul- 2016].

[10] "SimTK: Welcome", Simtk.org, (2016 )[Online]. Available: https://simtk.org [Accessed: 03- Jul2016].

[11] Chao, Y., et al., (2007), "Virtual Interactive Musculoskeletal System (VIMS) in OrthopedicResearch, Education and Clinical Patient Care," Journal of Orthopaedic Surgery and Research, pp. 1-19.

[12] "SIMM by MusculoGraphics, Inc.", Musculographics.com, (2016), [Online]. Available: http://www.musculographics.com [Accessed: 03- Jul- 2016]

[13] Delp SL, Anderson FC, Arnold AS, Loan P, Habib A, John CT, Guendelman E, Thelen DG, (2007)“'OpenSim: Open-source Software to Create and AnalyzeDynamic Simulations of Movement”, IEEE Transactions on Biomedical Engineering .

[14] F. GS, "Linking the mechanics and energetics of hopping with elastic ankle exoskeletons. - PubMed NCBI", Ncbi.nlm.nih.gov, (2016), [Online].Available: http://www.ncbi.nlm.nih.gov/pubmed/23065760. [Accessed: 02- Jul- 2016].

[15] Y.Tanjal, T.Nanjwa, Y.Takahashi, M. Kawal, (2016), "Evaluation of power assist System By Computer Simulation", JCII,Vol.20,No.3,pp.447-483.

[16] Kamran Shamaei, Massimo Cenciarini, and Aaron M. Dollar, (2011), "On the Mechanics of the Ankle in the Stance Phase of the Gait", 33rd Annual International Conference of the IEEE EMBS.

[17] Ajay Seth, Darryl Thelen, Frank C. Anderson, Scott L. Delp, (2005), "Model of trunk, pelvis and leg segments, 10 degrees of freedom and 18 muscles", Annals of Biomedical Engineering, vol.33, pp 829840.

[18] "How Scaling Works - OpenSim Documentation -", Simtk-confluence.stanford.edu, 2016. [Online]. Available:http://simtkconfluence.stanford.edu:8080/display/OpenSim/How+Scaling+Works\#HowScalingWorksScaling.[Accessed: 04- Jul- 2016].

[19] M. Margarida, (2013) "A multibody approach to the contact dynamics : a knee joint application", Hdl.handle.net. [Online]. Available: http://hdl.handle.net/1822/24564. [Accessed: 04Jun- 2016] 